\title{
Developing Electrochemical Impedance Immunosensor for the Detection of Myoglobin
}

\author{
Naga Siva Kumar Gunda ${ }^{1}$, Minashree Singh ${ }^{2}$, Susanta Sinha Roy ${ }^{1}$, and Sushanta K. Mitra ${ }^{1}$ \\ ${ }^{1}$ Micro and Nano-scale Transport Laboratory, Department of Mechanical Engineering, University of \\ Alberta, Edmonton, Alberta, Canada, T6G 2 G8. \\ Corresponding author's e-mail address: sushanta.mitra@ualberta.ca \\ ${ }^{2}$ Department of Pharmacy and Pharmaceutical Sciences, University of Alberta, Edmonton, Alberta, \\ Canada T6G 2 G8.
}

\begin{abstract}
:
In the present work, electrochemical impedance immunosensor is developed for detection of myoglobin. The procedure involves the immobilization of myoglobin antibodies on the interdigitated gold electrodes (IDE) using alkanethiol self-assembled monolayer (SAM) for binding myoglobin antigens available in the blood serum/aqueous solution. Then the sensor system is characterised using alternating current (AC) electrochemical impedance spectroscopy (EIS), in which the change in impedance spectra is observed across the frequency range of $0.1 \mathrm{~Hz}$ to $10 \mathrm{MHz}$. This work mainly focuses on understanding and improving the impedance immunosensor for better sensitivity readings $(\sim 1 \mathrm{ng} / \mathrm{mL})$ by adjusting the interdigitated electrode configuration, incubation times, reagent sample volumes and immobilisation protocols. Myoglobin is one of the premature indentifying cardiac protein markers to monitor minor heart attacks. Hence, the developed immunosensor has potential of using as a point-of-care diagnostic device. The protocol developed in this work can be useful for detecting other cardiac markers like Troponin and CK-MB by using respective antibodies.
\end{abstract}

Key words: electrochemical, impedance spectroscopy, immunosensor, myoglobin, self-assembled monolayer, fluorescent detection

\section{Introduction}

In recent years, impedance based direct immunosensors have made huge progress for the rapid detection of biomolecules. Electrochemical impedance spectroscopy is emerging as a reliable method for the analysis of interfacial property changes of modified electrode surfaces, which eventually used as bio-recognition event [1]. Impedance technique analyzes both the resistive and capacitive properties of the medium containing biomolecules and electrolytes. Impedancebased biosensors have been widely applied because of their high sensitivity and label-free operation. Motivated by the remarkable advantages, researchers are engaged to produce a variety of impedimetric biosensors to monitor various biochemical reactions at the surface of electrodes by immobilizing biomolecules such as enzymes, proteins, nucleic acids, etc. [2-8].

In this work, we fabricated gold interdigitated electrodes (IDEs) with various dimensions. IDEs were modified by alkanethiol selfassembled monolayers (SAMs) for the antibody immobilizations. Finally, alternating current (AC) impedance spectroscopic studies were performed after immobilization of antibody and the sensitivity of the immunosensors, at various IDEs dimensions were tested.

\section{Materials and Methods \\ Materials}

11-mercapto-1-undecanoic acid (MUA), 11mercaptoundecanoal (MU), Nhydroxysuccinimide (NHS) and 1-Ethyl-3-[3dimethylaminopropyl]carbodiimide

hydrochloride (EDC) were obtained from Sigma-Aldrich Chemicals, Ontario, Canada. Capture and detection myoglobin antibodies (antimyoglobin 7C3 and 4E2) were obtained from Hytest Ltd, Turku, Finland. Other chemicals (phosphate buffer saline (PBS), Sodium Chloride ( $\mathrm{NaCl})$, Ethanol, etc.) were of analytical grade and used without further purification.

Apparatus
Impedance measurements were performed
using VersaSTAT Impedance Analyzer
(VersaSTAT 4, Princeton Applied Research,


Oak Ridge, TN, USA) with VersaStudio software.

\section{Fabrication of Microfluidic Chip with Integrated} IDE's

First, IDE's were patterned on the glass substrate using standard photolithography process. Four different types of electrode configurations were used in the present work (Table 1). IDE is made-up of gold/chromium layer with $300 \mathrm{~nm}$ thickness. PDMS microchannels $(10 \mathrm{~mm}$ long, $2 \mathrm{~mm}$ width and 100 micron depth) with two reservoirs (inlet and outlet) were fabricated using soft-lithography process. Next, PDMS microchannel was aligned and bonded to IDE using oxygenplasma treatment. Microfluidic chip with integrated IDE is illustrated in Fig. 1.

Immobilization of Antibodies on Gold Electrode

Step1: Cleaning of Gold surface

Microfluidic chip with integrated IDE was cleaned in ethanol, isopropyl alcohol and deionized water and dried with nitrogen gas.

Step2: Preparation of alkanethiol selfassembled monolayer (SAM)

$4 \mathrm{mM}$ of MUA and $1 \mathrm{mM}$ of $\mathrm{MU}$ were prepared in ethanol and stored at room temperature. Then, IDE were immersed in a mixture of $4 \mathrm{mM}$ MUA / $1 \mathrm{mM}$ MU $(1: 1 ; \mathrm{v} / \mathrm{v})$ for overnight to obtain SAM. Then SAM prepared samples were washed in ethanol to remove unbound thiols and dried with nitrogen gas.

Step3: Preparation of EDC/NHS for antibody activation

$400 \mathrm{mM}$ of EDC and $100 \mathrm{mM}$ of NHS were prepared in freshly filtered Millipore deionized water, aliquoted and stored separately at $-20^{\circ} \mathrm{C}$. Then IDE were immersed in a mixture of 400mM EDC / 100mM NHS (1:1; v/v) for 10 minutes. This step helps to activate the sample surface to immobilize the antibodies.

Step4: Immobilization of Myoglobin antibodies

Capture myoglobin anitbodies were prepared in PBS at concentration of $100 \mu \mathrm{g} / \mathrm{mL}$. $2 \mu \mathrm{l}$ solution of myoglobin antibodies were injected into the PDMS channel and kept for $10 \mathrm{~min}$ incubation time. This step was used to immobilize the antibodies on IDE.

Step5: Testing of Antibody Immobilization

Antibody coated IDE was cleaned several times by injecting PBS buffer into the channels to test the performance of immobilization.

\section{Impedance measurements}

Figure 1 shows the experimental set-up arrangement for the impedance measurements. Electrochemical impedance measurements were performed using $1 \mathrm{M} \mathrm{NaCl}$ solution as an electrolyte without any redox species for different IDE sensors. For the impedance measurements, a sinuous modulated $A C$ potential of $10 \mathrm{mV}$ at frequency range of $0.1 \mathrm{~Hz}$ to $1 \mathrm{MHz}$ was applied across IDE. Bode plot was plotted for the magnitude (Ohms) and phase angle (deg) of impedance.

Tab. 1: Details of different IDEs used in this work

\begin{tabular}{|c|c|c|}
\hline $\begin{array}{c}\text { Type of } \\
\text { electrode } \\
\text { configuration }\end{array}$ & $\begin{array}{c}\text { Width of } \\
\text { electrode } \\
(\mu \mathrm{m})\end{array}$ & $\begin{array}{c}\text { Gap between } \\
\text { the } \\
\text { electrodes } \\
(\mu \mathrm{m})\end{array}$ \\
\hline IDE1 & 25 & 25 \\
\hline IDE2 & 25 & 50 \\
\hline IDE3 & 70 & 30 \\
\hline IDE4 & 75 & 25 \\
\hline
\end{tabular}

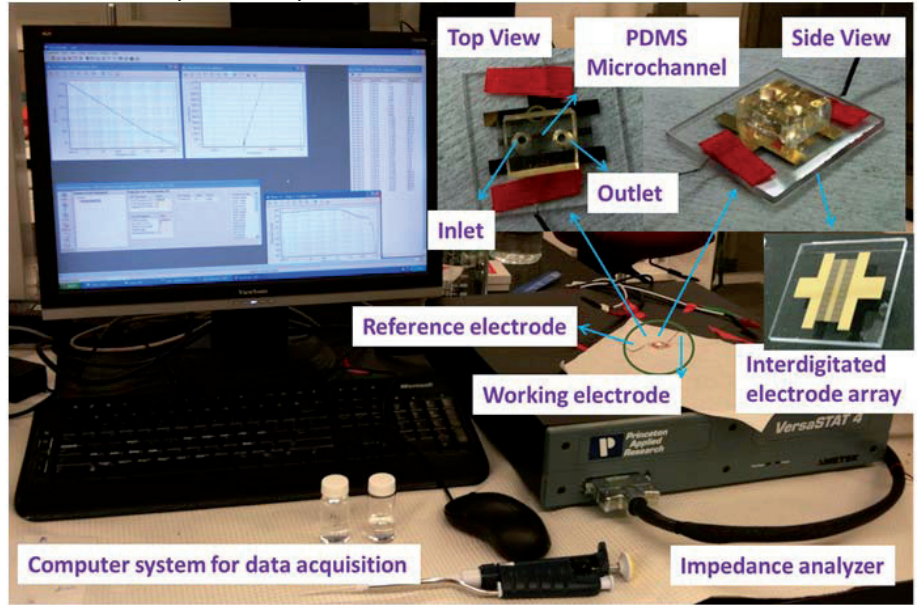

Figure 1.Experimental set-up for the impedance measurement with microfluidic chip with integrated IDE. 


\section{Results and Discussion}

The development of sensor protocol is characterized after each modification on the gold surface with ellipsometer, Fourier Transform Infrared Spectroscopy (FTIR), atomic force microscopy (AFM), and contact angle measurement system. This characterization identified the respective linked molecules produced after each step. Thickness of alkanethiol SAM is measured as $\sim 0.84 \mathrm{~nm}$ in ellipsometer. Since the main focus of the paper is to show the impedance spectra of IDEs at different steps, we have not presented the characterization results here.
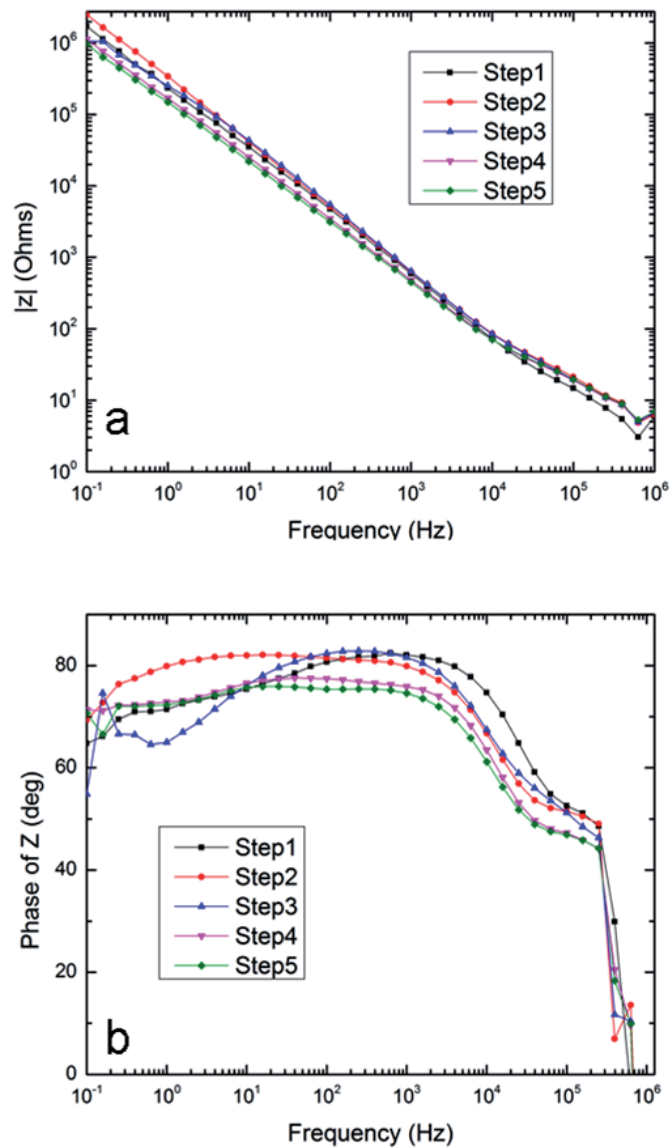

Fig. 2. Illustrations of Bode plot of impedance spectra for IDE3 after each step; (a) |Z| vs frequency; (b) the phase angle of $Z$ vs frequency.

We measured the impedance after each processes mentioned above. The whole frequency spectra showed a difference in both impedance and phase angle, possibly due to the change of double layer capacitance $\left(C_{d l}\right)$ and charge transfer resistance $\left(R_{c t}\right)$. Bode plot can be useful to understand the characteristics frequency range of the sensors. Two representative impedance spectra are shown in Fig. 2 and Fig. 3. Figure 2 shows the effect of each process on the impedance spectra and Fig. 3 shows the effect of electrode dimensions on the impedance spectra. The results shown in Figs. 2 and 3 are matches with the existing literature results on impedimetric sensors.

In the present work, we used $1 \mathrm{M} \mathrm{NaCl}$ solution as electrolyte without any redox species, whereas most other reports considered $\left[\mathrm{Fe}(\mathrm{CN})_{6}\right]^{-3 /-4}$ as redox species. Redox species increases Faradaic reaction, which will increase the insulation of surface. This can be easily detected by change in resistance $\left(R_{c t}\right)$. In nonFaradaic case (the present study), impedance spectra is dominated by equivalent capacitance change in the set-up due to double layer formation at the electrode surfaces.
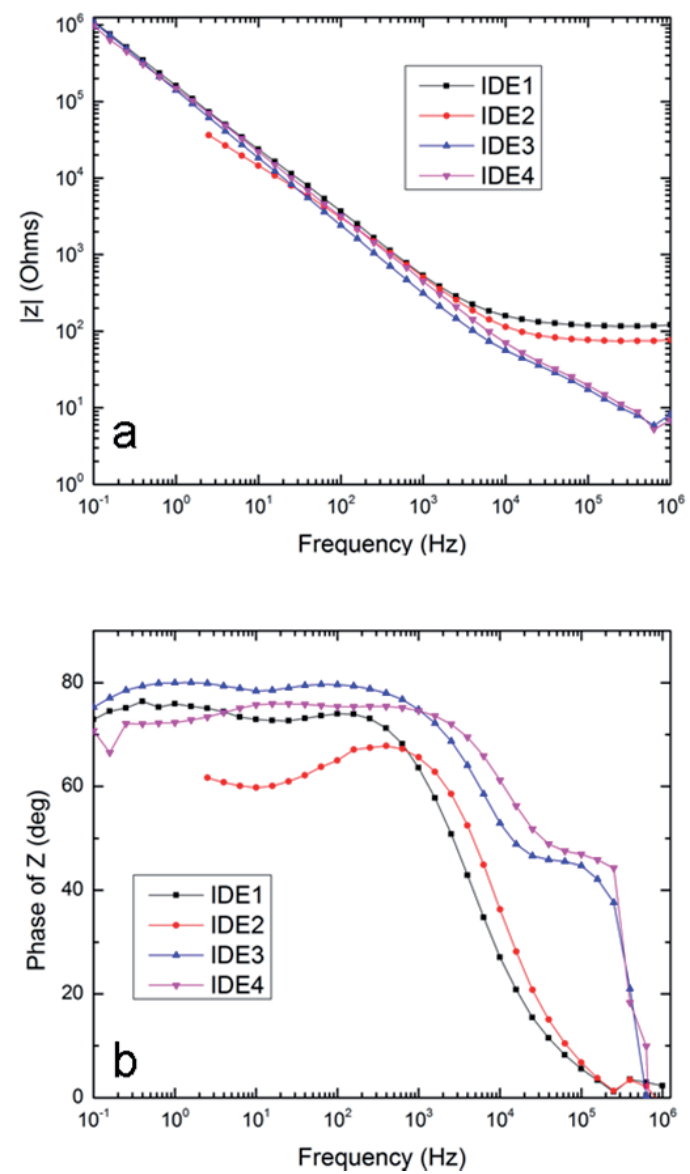

Fig. 3. Illustrations of Bode plot of impedance spectra for various IDEs after step 5; (a) |Z| vs frequency; (b) the phase angle of $Z$ vs frequency.

These Bode plots are used to decide the working frequency range to study the performance of sensor. At higher frequencies, the increase in electrode area is the dominant 
parameter for change in impedance as observed in Fig. 3a. From the results (Figs. 2a and $3 \mathrm{a}$ ), it is observed that $10 \mathrm{~Hz}$ to $10 \mathrm{KHz}$ is the frequency range where one can observe a large change in impedance.

\section{Conclusion}

The present work discussed the methodology used to develop electrochemical impedance immunosensor for myoglobin detection. The reagent volume and incubation times were reduced by using microchannels. In future, we will study the effects of electrode size, gap size, and thickness of electrode.

\section{Acknowledgements}

We acknowledge Dr. Marc Secanell and his Ph.D student Shantanu Shukla, Energy Systems Design Laboratory, Department of Mechanical Engineering, University of Alberta for allowing us to use some of the facilities to carry out our experiments. We also gratefully acknowledge the financial support of 'Alberta Innovates - Technology Futures' in the form of scholarships for NSKG.

\section{References}

[1] C.Berggren, B. Bjarnason, and G. Johansson, Capacitive Biosensors, Electroanalysis 13 (3), 173-180 (2001).

[2] S. Grant, F. Davis, K. A. Law, A. C. Barton, S. D. Collyer, S. P. J. Higson and T. D. Gibson, Label-free and reversible immunosensor based upon an ac impedance interrogation protocol, Anal. Chim. Acta. 537, 163-168 (2005)

[3] E. Katz and I. Willner, Probing Biomolecular Interactions at Conductive and Impedimetric Immunosensors, DNA-Sensors, and Enzyme Biosensors, Electroanalysis, 15, 913-937 (2003).

[4] E. Bakker and Y. Qin, Electrochemical sensors, Anal. Chem. 78, 3965-3984 (2006).

[5] M. A. Rahman, M. J. A. Shiddiky, J. Park and Y. Shim, An impedimetric immunosensor for the label-free detection of bisphenol A, Biosens. Bioelectron. 22, 2464 (2007)

[6] E. M. Hamad, Impedimetric Sensors for Label-Free Point-of-care Immunoassay Cardiac Marker Systems, Ph.D thesis, University of Ulster, January 2011.

[7] M. Hnaieni, M. F. Diouani, S. Helali, L. Hafaid, W.M. Hassen, N. J. Renault, A. Ghram and A. Abdelghani, Immobilisation of specific antibody on SAM functionalized gold electrode for rabies virus detction by electrochemical impedance spectroscopy, Biochemical Engineering Jounral 39 (3) 443-449 (2008).

[8] A. C. Barton, S. D. Collyer, F. Davis, G. Garifallou, G. Tsekenis, E. Tully, R. O Kennedy, T. Gibson, P. A. Millner and S. P.J, Labless, AC impedimetric antibodybased sensors with $\mathrm{pg} / \mathrm{m}$; sensitivities for point-of-care biomedical applications, Biosensors and Bioelectrornics 24, (25), Jan 2009.

[9] N. S. K. Gunda, S. K. Mitra and V. R. Rao, Proceedings of the Seventh International ASME Conference on Nanochannels, Microchannels and Minichannels, ICNMM09, Pohang, South Korea, June 22-24 (2009). 